International Journal of Pure and Applied Mathematics

Volume 106 No. 3 2016, 873-881

ISSN: 1311-8080 (printed version); ISSN: 1314-3395 (on-line version)

url: http://www.ijpam.eu

doi: 10.12732/ijpam.v106i3.13

ijpam.eu

\title{
ON THE DYNAMICS OF A FAMILY OF RATIONAL FUNCTIONS WHICH CONTAINS KOEBE FUNCTION
}

\author{
Kuldeep Prakash ${ }^{1}$, R.P. Pant ${ }^{2}$ \\ ${ }^{1,2}$ Department of Mathematics \\ D.S.B. Campus \\ Kumaun University \\ Nainital, INDIA
}

\begin{abstract}
We study the dynamics of a parameterized family of quadratic rational functions $z \mapsto \lambda z /(1-z)^{2}, \lambda \in(0, \infty)$. We have investigated the dynamical and geometric properties of Julia sets and the nature of the variance of these properties according to the parameter. We have shown the Julia sets are connected when $\lambda \geqslant 1$ and are dynamically defined cantor sets when $\lambda<1$. We also have shown the continuity of geometric properties specially in terms of Hausdorff dimensions of these Julia sets.
\end{abstract}

AMS Subject Classification: $37 \mathrm{~F}$

Key Words: Julia sets, Hausdorff dimension, bifurcation, Cantor sets

\section{Introduction}

In this paper we consider the family

$$
K_{\lambda}(z)=\frac{\lambda z}{(1-z)^{2}} .
$$

When $\lambda=1$, we get the famous Koebe function. Koebe function is most celebrated function in geometric function theory. It is instrumental in proving many important results. However from dynamical point of view the function

Received: November 28, 2015

Published: February 27, 2016

${ }^{\S}$ Correspondence author (c) 2016 Academic Publications, Ltd.

url: www.acadpubl.eu 
is not explored much. Stienmetz [11] has discussed the Koebe function in unsolved exercises of his book. Here we describe the complete dynamics of a larger family of rational maps containing Koebe function as a member and show the continuity of the Julia sets and their Hausdorff dimensions.

Two rational maps $R$ and $S$ are said to be conjugate if there is a mobius transformation $g$ such that $S=g R g^{-1}$. It is a well known fact that two conjugate maps are dynamically equivalent to each other. It can easily be seen that the results obtained for the family $K_{\lambda}$ are also valid for the families containing the maps $z \mapsto z /(\lambda-z)^{2}$ and $z \mapsto z /(1-\lambda z)^{2}$ where $\lambda$ is taken in $(0, \infty)$.

A lot of work has been done on the dynamics of quadratic rational maps. Milnor [7, 8] and Rees [9] have investigated the moduli space of quadratic rational maps in general. Particular slices of this moduli space have also been studied by Hawkins [4] and Milnor [7].

The concept of conformal measures is one of the main tools to understand fractal properties of a Julia set, i.e. its dimensions and measures. The concept of conformal measures was first employed to the case of rational functions by Sullivan in [12]. Basic results concerning conformal measures, Hausdorff dimension and fractal and ergodic properties of Julia sets of rational functions of the Riemann sphere can be found in a survey done by Urbanski [13].

\section{Preliminaries}

The Fatou set $F(f)$ of a function $f$ is defined as the set of those points $z \in \widehat{\mathbb{C}}$ that has an open neighborhood $U$ such that the family of iterates $\left\{f^{n}(U)\right\}_{n \in \mathbb{N}}$ is normal with respect to some spherical metric on $\overleftrightarrow{\mathbb{C}}$. The Julia set $J(f)$ is then defined as $\widehat{\mathbb{C}}-F(f)$. A remarkable Theorem of Montel, which paved the way for Fatou and Julia to develop a comprehensive global theory of iterates of rational functions, is as follows:

Theorem 1. Let $\mathcal{F}$ be a family of meromorphic functions defined on a domain $U$. Suppose there exist points $a, b, c$ in $\widehat{\mathbb{C}}$ such that $\left[\bigcup_{f \in \mathcal{F}} f(U)\right] \cap$ $\{a, b, c\}=\phi$. Then $\mathcal{F}$ is a normal family on $U$.

The following basic properties of Julia sets may be found in [1] and [6]:

1. Julia set of a function $f, J(f)$ is a non-empty compact subset of $\widehat{\mathbb{C}}$.

2. $J(f)$ is totally invariant, i.e. $f^{-1}(J(f))=f(J(f))=J(f)$.

3. Repelling periodic points of $f$ are dense in $J(f)$. 
Given a nonnegative real number $t$, t- dimensional Housdorff measure $H_{t}(A)$ of a set $A$ is defined as

$$
H_{t}(A)=\sup _{\epsilon>0} \inf \left\{\sum_{i=1}^{\infty}\left(\operatorname{diam}\left(A_{i}\right)\right)^{t}\right\}
$$

where the infrimum is taken over all countable covers $\left\{A_{i}: i \geqslant 1\right\}$ of $A$ by arbitrary sets whose diameters do not exceed $\epsilon$. The Hausdorff dimension $\operatorname{HD}(A)$ of $A$ is defined as,

$$
\mathrm{HD}(A)=\inf \left\{t: \mathrm{H}_{t}(A)=0\right\}=\sup \left\{t: \mathrm{H}_{t}(A)=\infty\right\}
$$

Along with the Hausdorff dimension there are some other notions which have been frequently employed to visualize the fractal structures of Julia sets. Here we recall some of them which we shall use in the proofs

1. The Poincaré series is defined as,

$$
\Xi_{s}(f, z)=\sum_{n=0}^{\infty} \sum_{f^{n}(\zeta)=z} \frac{1}{\left|\left(f^{n}\right)^{\prime}(\zeta)\right|^{s}}
$$

2. The critical exponent at $z$ by

$$
\delta_{c r}(f, z)=\inf \left\{s>0: \Xi_{s}(f, z)<\infty\right\}
$$

and critical exponent of $f$ by

$$
\delta_{c r}(f)=\inf _{\mathbb{C}} \delta_{c r}(f, z)
$$

3. Let $t \geqslant 0$. A measure $m$ on $J(f)$ is said to be a Conformal Measure of exponent $t$ for $f: \widehat{\mathbb{C}} \rightarrow \widehat{\mathbb{C}}$ if $m(J(f))=1$ and

$$
m(f(A))=\int_{A}\left|f^{\prime}\right|^{t} d m
$$

for every Borel set $A \subset J(f)$ such that $\left.f\right|_{A}$ is injective map. Moreover minimal possible exponent for which a conformal measure exists for $f$ will be denoted by $\delta(f)$. 
It is well known that for a geometrically finite map $f$ the Hausdorff dimension of the Julia set $\operatorname{HD}(J(f))$ is equal to $\delta(f)$.

We define $\operatorname{Comp}(\widehat{\mathbb{C}})$ as the space of all non empty compact sets in $\widehat{\mathbb{C}}$ with the Hausdorff topology. We recall from [5] that in the Hausdorff topology, If $\left\{X_{n}\right\}$ is a sequence of subsets of a space $S$. The set of all points $x$ in $S$ such that every open set containing $x$ intersects all but a finite number of the sets $X_{n}$ is called the limit inferior of the sequence $\left\{X_{n}\right\}$ and is abbreviated "lim inf $X_{n}$ "; the set of all points $y$ in $S$ such that every open set containing $y$ intersects infinitely many sets $X_{n}$ is called the limit superior of $\left\{X_{n}\right\}$ and is abbreviated "lim $\sup X_{n}$." If these two sets coincide so that $\lim \inf X_{n}=L=\lim \sup X_{n}$, we say that $\left\{X_{n}\right\}$ is a convergent sequence of sets and that $L$ is the limit of $\left\{X_{n}\right\}$, which is abbreviated " $L=\lim X_{n}$."

\section{Dynamics of the maps}

Theorem 2. The Julia set $J\left(K_{\lambda}\right)=J_{\lambda}$ of the map $K_{\lambda}$ is the set of all nonnegative real numbers with infinity if $\lambda \geqslant 1$.

$$
J_{\lambda}=[0, \infty]
$$

Proof. First we shall show that family $\left\{K_{\lambda}^{n}\right\}_{n}$ is normal in $F=\widehat{\mathbb{C}}-[0, \infty]$. We note that if $K_{\lambda}(z) \notin F$ then $z \notin F$, For let $K_{\lambda}(z)=r$ where $r$ is nonnegative real number then $z$ satisfies,

$$
r z^{2}-(2 r+\lambda) z+1=0 \quad \text { or } \quad z=\frac{(2 r+\lambda) \pm \sqrt{\lambda^{2}+4 r \lambda}}{2 r}
$$

Thus $z$ is a nonnegative real number so $K_{\lambda}(F)=F$. Using this fact with Montel Theorem the family $\left\{K_{\lambda}^{n}\right\}_{n}$ is normal in $F, F$ is contained in the Fatou set $F\left(K_{\lambda}\right)=F_{\lambda}$.

Next $0 \in J_{\lambda}$. For if $0 \in F_{\lambda}$ then there exist an open neighborhood of $0, F_{0}$ which is contained in the Fatou set $F_{\lambda}$. let us take,

$$
F^{\prime}=F_{\lambda} \cap[0, \infty]
$$

then $F_{0} \cap[0, \infty] \subset F^{\prime}$ and because $F_{0}$ is open there exist $\varepsilon>0$ such that $(0, \varepsilon) \subset F^{\prime}$. But since 0 is fixed point and $K_{\lambda}(\varepsilon)>\varepsilon$ when $\varepsilon<1$ there exist $n \in \mathbb{Z}$ such that $[0,1] \subset K_{\lambda}^{n}[0, \varepsilon)$ using the fact that Fatou sets of rational maps are completely invariant and $K_{\lambda}[0,1]=[0, \infty]$, we have $[0, \infty] \subset F^{\prime} \subset F_{\lambda}$ and thus $F_{\lambda}=\mathbb{C}$ which is not possible since Julia set of a rational map of degree greater then 2 is nonempty. 
To prove $J_{\lambda}=[0, \infty]$ it remains to prove $F^{\prime}=\phi$. Since $K_{\lambda}(\infty)=0, \infty \notin F_{\lambda}$ so $F^{\prime}$ is open set in $\mathbb{R}$. We see $d\left(0, F^{\prime}\right) \neq 0$, for if $d\left(0, F^{\prime}\right)=0$ then because $F^{\prime}$ is open set there exist $\varepsilon>0$ such that $(0, \varepsilon) \subset F^{\prime}$ which leads to $\infty \in F_{\lambda}$ as before, a contradiction. So $d\left(0, F^{\prime}\right) \neq 0$ and therefore there exist $\delta>0$ such that, $(0, \delta) \cap F^{\prime}=\phi \Longrightarrow(0, \delta) \cap F_{\lambda}=\phi \Longrightarrow(0, \delta) \subset J_{\lambda}$. Since $J_{\lambda}$ is completely invariant, $K_{\lambda}^{n}(0, \delta) \subset J_{\lambda}$ for every $n \in \mathbb{Z}$. We have noted above that there exist $n \in \mathbb{Z}$ such that $[0, \infty] \subset K_{\lambda}^{n}(0, \delta)$, so $[0, \infty] \subset J_{\lambda}$ this with the fact $F_{0}=\widehat{\mathbb{C}}-[0, \infty]$ completes the proof.

Theorem 3. The Julia set of $K_{\lambda}$ is a cantor set which is contained in $[1-\sqrt{\lambda}, 1 /(1-\sqrt{\lambda})]$ when $\lambda<1$.

Proof. As in Theorem 2 we can see that the Fatou set $F_{\lambda}$ contains $F=$ $\widehat{\mathbb{C}}-[0, \infty]$ this implies Fatou set is connected. From this and the fact 0 is attractive fixed point we can deduce

$$
K_{\lambda}^{n}(z) \rightarrow 0 \quad \text { when } \quad z \in F_{\lambda}
$$

We now begin our construction of Julia set. first we observe that $K_{\lambda}(z)<z$ if $z \in(0,1-\sqrt{\lambda})$ and $K_{\lambda}(1 /(1-\sqrt{\lambda}), \infty)=(0,1-\sqrt{\lambda})$. Thus $K_{\lambda}^{n}(z) \rightarrow 0$ if $z \notin[1-\sqrt{\lambda}, 1 /(1-\sqrt{\lambda})]$. So,

$$
J_{\lambda} \subset J=[1-\sqrt{\lambda}, 1 /(1-\sqrt{\lambda})]
$$

Now we define $R_{1}, R_{2}:(0, \infty) \rightarrow(0,1)$ such that,

$$
R_{1}(w)=\frac{(2 w+\lambda)-\sqrt{\lambda^{2}+4 w \lambda}}{2 w}
$$

and

$$
R_{2}(w)=\frac{(2 w+\lambda)+\sqrt{\lambda^{2}+4 w \lambda}}{2 w} .
$$

We consider the images of $J$ under the elements of semi group containing maps generated by $R_{1}$ and $R_{2}$. So for every sequence of integers $i_{n}$ in $\{1,2\}$, we define: $J\left(i_{1}, \ldots, i_{m}\right)=R_{i_{1}} \ldots R_{i_{m}}(J)$ It can be easily seen that $J\left(i_{1}, \ldots, i_{m}, i_{m+1}\right) \subset$ $J\left(i_{1}, \ldots, i_{m}\right)$. Since the maps $R_{1}$ and $R_{2}$ are homeomorphisms onto two disjoint sets, the $2^{m}$ sets of the form $J\left(i_{1}, \ldots, i_{m}\right)$ are pairwise disjoint compact sets,

$$
K_{\lambda}^{-m}(J)=\bigcup_{i_{1}, \ldots, i_{m}=1}^{2} J\left(i_{1}, \ldots, i_{m}\right)
$$


We claim that the Julia set,

$$
J_{\lambda}=\bigcap_{m=0}^{\infty} K_{\lambda}^{-m}(J)
$$

For, by (2) and complete invariance of $J_{\lambda}$ we get, $J_{\lambda} \subset \bigcap_{m=0}^{\infty} K_{\lambda}^{-m}(J)$. And if $z \in \bigcap_{0}^{\infty} K_{\lambda}^{-m}(J)$ then $K_{\lambda}^{n}(z) \in J$ for all $n$. So $K_{\lambda}^{n}(z) \nrightarrow 0$ and by $(1) z \notin F_{\lambda}$, yeilding $\bigcap_{m=0}^{\infty} K_{\lambda}^{-m}(J) \subset J_{\lambda}$.

By construction it is clear that $J_{\lambda}$ is a compact set. To prove $J_{\lambda}$ is cantor set we only have to show that it is totally disconnected. If $J^{\prime}$ is any connected subset of $J_{\lambda}$, then for some $n$ it is contained in one and only one of the sets of the form $J\left(i_{1}, \ldots, i_{n}\right)$, so we have a unique sequence $\left(k_{n}\right)$ such that

$$
J^{\prime} \subset \bigcap_{n=1}^{\infty} J\left(k_{1}, \ldots, k_{m}\right)
$$

To prove $J^{\prime}$ is totally disconnected, we shall show $J^{\prime}$ is singleton set or equivalently

$$
\operatorname{diam}\left[J\left(k_{1}, \ldots, k_{m}\right)\right] \rightarrow 0 \quad \text { as } \quad m \rightarrow \infty
$$

For $z \in J$ we have, $\left|\left(K_{\lambda}^{n}\right)^{\prime}(z)\right|_{\sigma} \geqslant\left(\frac{2}{\sqrt{\lambda}}-1\right)^{n}$, where $\sigma$ is chordal metric on Riemann sphere. Chordal and Euclidean metrics are comparable on compact set $J$, this together with the fact $K_{\lambda}\left(R_{2}(z)\right)=K_{\lambda}\left(R_{1}(z)\right)=z$ yields,

$$
\begin{gathered}
\operatorname{diam}\left[J\left(k_{1}, \ldots, k_{m}\right)\right]<\frac{C}{\left(\frac{2}{\sqrt{\lambda}}-1\right)^{m}} \quad \text { for some } C \\
\quad \operatorname{diam}\left[J\left(k_{1}, \ldots, k_{m}\right)\right] \rightarrow 0 \quad \text { as } \quad m \rightarrow \infty
\end{gathered}
$$

Corollary 4. The map $K_{\lambda}$ restricted to its Julia set $J_{\lambda}$ is topologically conjugate to shift map $\sigma$ in $\{1,2\}^{\mathbb{N}}$.

Proof. From the proof of the theorem it is clear that for a given $x$ in $J_{\lambda}$ and for each $n \in \mathbb{N}$ there exist a unique $i_{n} \in\{1,2\}$ such that $x \in J\left(i_{1}, \ldots, i_{n}\right)$. To every $x \in J$ we can associate a sequence $\theta_{x}=i_{1}, i_{2}, \ldots, i_{n}, \ldots$, then $\theta_{K_{\lambda}(x)}=$ $i_{2}, i_{3}, \ldots, i_{n}, \ldots$ We define $f: J_{\lambda} \rightarrow\{1,2\}^{\mathbb{N}}$, such that $f(x)=\theta_{x} . f$ is required conjugacy. 
Now, we investigate the continuity of Julia sets with respect to the parameter $\lambda$. It is well-known that the Julia set may vary discontinuously at some points. Parabolic implosions for quadratic polynomials is studied in [13]. Parabolic implosion refers to the phenomenon of discontinuity that sometimes appear at parabolic bifurcations. So it is particularly useful to investigate the continuity of Julia set at $\lambda=1$, since it is a parabolic bifurcation point. Here we demonstrate with relatively simple arguments that Julia set does depend continuously on the parameter even at the parabolic bifurcation in the case of family taken by us. We use some arguments of Douady [3] in the first part of the proof of the following theorem

Theorem 5. Julia sets $J_{\lambda}$ continuously depend on $\lambda$

Proof. We shall prove that the function $f:[0, \infty] \rightarrow \operatorname{Comp}(\widehat{\mathbb{C}})$ such that $f(\lambda)=J_{\lambda}$ is a continuous function or equivalently $J_{\lambda_{n}} \rightarrow J_{\lambda}$ whenever $\lambda_{n} \rightarrow \lambda$.

It is obvious when $\lambda \geqslant 1$. So let us take a sequence $\left(\lambda_{n}\right)$ in $(0,1)$ such that $\lambda_{n} \rightarrow \lambda \in(0,1]$. First we shall show that $J_{\lambda} \subset \lim \inf J_{\lambda_{n}}$. For let $x \in J_{\lambda}$ and $\mathrm{U}$ be some neighborhood of $x$ then since Julia set is closure of the set of repelling periodic points there exist a repelling point $y \in U$. This $y$ is a simple solution of the equation $K_{\lambda}{ }^{k}(z)-z=0$ for some $k \in \mathbb{N}$. By the implicit function theorem there exist $m>0$ such that $K_{\lambda_{n}}$ has a repelling periodic point of order $k$ in $U$ whenever $n \geq m$ implying that $J_{\lambda_{n}} \cap U=\phi$ whenever $n \geq m$. Thus $J_{\lambda} \subset \lim \inf J_{\lambda_{n}}$.

To prove $J_{\lambda_{n}} \rightarrow J_{\lambda}$, we only have to show lim sup $J_{\lambda_{n}} \subset J_{\lambda}$. Now let us assume $x \in F_{\lambda}=\mathbb{C}-J_{\lambda}$. We have to show that there exist a neighborhood of $x$ which is contained in all but finitely many $F_{\lambda_{n}}$. Since Fatou set is completely invariant, it is sufficient to show it for any iterate $K_{\lambda}^{i}(x)$ in place of $x$. We have already seen that $J_{\lambda_{n}} \subset\left[1-\sqrt{\lambda_{n}}, 1 /\left(1-\sqrt{\lambda_{n}}\right)\right]$. So there exist $m \in \mathbb{N}$ and a neighborhood $U$ of 0 such that $U \subset F_{\lambda_{n}}$ for all $n \geq m$. But since 0 is attractive fixed point when $\lambda \in(0,1)$ and $x \in F_{\lambda}$, we have $K_{\lambda}{ }^{i}(x) \in U$. So lim $\sup J_{\lambda_{n}} \subset J_{\lambda}$. When $\lambda=1$ it is obvious since $F_{1}=\widehat{\mathbb{C}}-[0, \infty] \subset F_{\lambda}$ for every $\lambda$. Hence $J_{\lambda_{n}} \rightarrow J_{\lambda}$.

\section{Hausdorff dimensions of Julia sets}

Theorem 6. If $\lambda_{n} \rightarrow \lambda$ then $\operatorname{HD}\left(J\left(K_{\lambda_{n}}\right)\right) \rightarrow H D\left(J\left(K_{\lambda}\right)\right)$

Proof. There is nothing to prove when $\lambda>1$. When $\lambda<1, K_{\lambda}$ and $K_{\lambda_{n}}$ are expansive maps. Real analyticity of Housdorff dimensions for hyperbolic maps 
has been shown in [10]. So it only remains to be shown in the case $\lambda=1$ and $\lambda_{n}<1$.

We have seen in the Theorem 4 that $J_{\lambda_{n}} \rightarrow J_{\lambda}$ in the Hausdorff topology. It is easy to see $K_{\lambda_{n}}$ are the hyperbolic maps. So theorem 2.3 of Urbanski ensures the existence of an unique conformal measure of exponent $\operatorname{HD}\left(J_{\lambda_{n}}\right)$.

Since $J_{\lambda_{n}}$ are cantor sets contained in $\mathbb{R}$. we have $\operatorname{HD}\left(J_{\lambda_{n}}\right)<1$. So lim sup $\operatorname{HD}\left(J_{\lambda_{n}}\right) \leqslant 1$. And we know from that

$$
H D\left(J_{\lambda_{n}}\right)=\delta\left(K_{\lambda_{n}}\right)
$$

Let $\delta\left(K_{\lambda_{n}}\right)$ be the exponents of conformal measures $\mu_{\lambda_{n}}$ Now let $\lim \inf \delta\left(K_{\lambda_{n}}\right)=$ $\delta_{0}$ than we consider the subsequence $\delta\left(K_{\lambda_{n}}\right)$ convergent to $\delta_{0}$ and such that normalized conformal measures $\mu_{\lambda_{n}}$ of dimension $\delta\left(K_{\lambda_{n}}\right)$ converge weakly to a measure $\mu$. Since $J_{\lambda_{n}} \rightarrow J_{1}, \mu$ is a conformal measure for $K_{1}$ on $\mathbb{C}$. If $\mu$ is supported on Julia set $J_{1}$ then $\delta_{0} \geqslant \delta\left(K_{1}\right)=1$. If not, support of $\mu$ contains some $z \in F_{1}$. Since Koebe function is locally univalent on $F_{1}$ there exist a ball $B$ centered at $\mathrm{z}$ such that all the preimages of $B$ are disjoint. By this along with Koebe distortion theorem we can deduce that $\mu\left(\bigcup K_{1}{ }^{-n}(B)\right)$ is finite. Since $\Xi_{\delta_{0}}\left(K_{1}, z\right)$ is comparable to $\mu\left(\bigcup K_{1}^{-n}(B)\right)$, it is also finite and $\delta_{c r}\left(K_{\lambda}, z\right) \leqslant \delta_{0}$. Now we follow Sullivan [12] construction of conformal measures for general rational maps to show that there exist a conformal measure of exponent $\delta_{c r}\left(K_{\lambda}, z\right)$ supported on the Julia set $J_{1}$. Let us define,

$$
\left.m_{s}=\frac{1}{\Xi_{s}\left(K_{1}, z\right)} \sum_{K_{1}^{n}(w)=z} \mid K_{1}^{n}\right)\left.^{\prime}(w)\right|^{-s} \delta_{w}
$$

where $s$ is taken greater then $\delta_{c r}\left(K_{1}, z\right)$ and $\delta_{w}$ is $\delta$ mass at $w$.

Now let $A$ be any Borel set and $\left.K_{1}\right|_{A}$ is injective map then using chain rule we have

$$
\int_{A}\left|K_{1}^{\prime}\right|^{s} d m_{s}= \begin{cases}m_{s}\left(K_{1}(A)\right) & \text { if } z \notin A \\ m_{s}\left(K_{1}(A)\right)+\left|K_{1}^{\prime}(z)\right|^{s} / \Xi_{s}\left(K_{1}, z\right) & \text { if } z \in A\end{cases}
$$

If $\Xi_{s}\left(K_{1}, z\right)$ is divergent at $s=\delta_{c r}\left(K_{1}, z\right)$. we define $m$ as a weak limit of $m_{s}$ as $s \rightarrow \delta_{c r}(f, z)$. So in this case we get

$$
\int_{E}\left|K_{1}^{\prime}\right|^{t} d m=m(f(A))
$$

where $t=\delta_{c r}\left(K_{1}, z\right)$. If $\Xi_{s}\left(K_{1}, z\right)$ is convergent at $s=\delta_{c r}\left(K_{1}, z\right)$. Then we shall modify our definition of $m_{s}$ by introducing new weighting factors 
$\left.\left.\left.h\left(\mid K_{1}^{n}\right)^{\prime}(w)\right|^{-1}\right) \mid K_{1}^{n}\right)\left.^{\prime}(w)\right|^{-s}$ for masses placed along $\left\{w \mid f^{n}(w)=z\right\}$ to define $m_{s}$. where function $h:(0, \epsilon) \rightarrow(0,+\infty)$ such that $\lim _{t \rightarrow 0}(h(a t) / h(t))=1$ for every $a>0$ and $\left.\left.\left.h\left(\mid K_{1}^{n}\right)^{\prime}(w)\right|^{-1}\right) \mid K_{1}^{n}\right)\left.^{\prime}(w)\right|^{-\delta}=\infty$. This completes the proof of the existence of conformal measures on any Julia set.

Thus $\delta_{c r}\left(K_{1}, z\right) \geqslant \delta\left(K_{1}\right)$. Since $K_{1}$ is geometrically finite $\delta\left(K_{1}\right)=\operatorname{HD}\left(K_{1}\right)=$ 1 . So $1 \leqslant \delta_{c r}\left(K_{1}, z\right) \leqslant \delta_{0}$. So in each case,

$$
1 \leqslant \lim \inf \delta\left(K_{n}\right)=\lim \inf \left(\operatorname{HD}\left(J_{\lambda_{n}}\right)\right)
$$

$\lim \inf \left(\operatorname{HD}\left(J_{\lambda_{n}}\right)\right)=\lim \sup \left(\operatorname{HD}\left(J_{\lambda_{n}}\right)\right)=1$.

Hence, $\operatorname{HD}\left(J\left(K_{\lambda_{n}}\right)\right) \rightarrow \operatorname{HD}\left(J\left(K_{\lambda}\right)\right)$.

\section{References}

[1] L. Carleson, T. Gamelin, Complex Dynamics, Springer- Verlag, (1993).

[2] A. Douady, Does a Julia set continuously depend on the polynomials ?, Complex Analytic Dynamics, Amer. Math. Soc., (1994).

[3] A. Douady, P. Sentenac, M. Zinsmeister, Implosion Parabolique et dimension de Hausdorff, C. R. Acad. SCi. Paris Ser. I Math, 325(1997), 765-772.

[4] J. Hawkins, Lebesgue ergodic rational maps in parameter space. Int. J. Bifurcation and Chaos, 13 (2003), no. 6, 1423-1447.

[5] J. G. Hocking, G. S. Young. Topology, Addison- Wesley, (1961).

[6] J. Milnor, Dynamics in One Complex Variable, (AM-160), Princeton University Press, (2011)

[7] J. Milnor, Geometry and dynamics of quadratic rational maps, Experiment. Math., 2 (1993), no. 1, 37-83.

[8] J. Milnor, On rational maps with two critical points, Experiment. Math., 9 (2000), no. 4, 481-522.

[9] M. Rees, Components of degree two hyperbolic rational maps. Invent. Math., 100 (1990), no. 2, 357-382.

[10] D. Ruelle, Repellers for real analytic maps, Ergodic Theory Dynamical Systems, 2 (1982), 99-108.

[11] N. Steinmetz, Rational Iteration, de Gruyter Studies in Mathematics 16, (1993).

[12] D. Sullivan, Conformal dynamical systems, Lecture Notes in Mathematics, 1007 Springer Verlag (1983), 725-752.

[13] M. Urbanski, Measures and dimensions in conformal dynamics, Bulletin of the American Mathematical Society 40.3 (2003), 281-321. 
